2. David Kleinecke, Almost-finite, compact and inessential operators, Proc. Amer. Math. Soc. 14 (1963), 863-868.

3. S. R. Caradus, Operator of Riesz type, Pacific J. Math. 18 (1966), 61-71.

4. I. C. Gohberg and M. G. Krein, Fundamental aspects of defect numbers, root numbers, and indices of linear operators, Uspehi Mat. Nauk. 12 (1957), 43-118.

5. Shmuel Kaniel and Martin Schechter, Spectral theory for Fredholm operators, Comm. Pure Appl. Math. 16 (1963), 423-448.

6. I. A. Feldman, I. C. Gohberg and A. S. Markus, Normally solvable operators and ideals associated with them, Izv. Moldavsk Filiala Akad. Nauk. SSSR, 10 (1960), 51-69.

7. Bertram Yood, Difference algebras of linear transformations on a Banach space, Pacific J. Math. 4 (1954), 615-636.

8. - Properties of linear transformations preserved under addition of a completely continuous transformation, Duke Math. J. 18 (1951), 599-612.

9. A. F. Ruston, Operators with Fredholm theory, J. London Math. Soc. 29 (1954), 318-326.

Belfer Graduate School of Science, Yeshiva University

\title{
THE CENTRALIZER OF A REGULAR UNIPOTENT ELEMENT IN A SEMISIMPLE ALGEBRAIC GROUP
}

\author{
BY BETTY LOU 1
}

Communicated by Louis Auslander, June 14, 1968

The following question was posed by Springer [2]: is the centralizer $G_{x}$ of a regular unipotent element $x$ in a semisimple algebraic group $G$ abelian? In this paper we shall give an affirmative answer and also find the number of disjoint components of $G_{x}$ if it is reducible. The problem is easily reduced to the case in which $G$ is simple, which we henceforth assume. As proved by Springer in [2], reducibility occurs only when the type of $G$ and the characteristic $p$ of the base field $\Phi$ are related as follows: $C_{n}(n \geqq 2)$ and $D_{n}(n \geqq 4)$ with $p=2$ (here $B_{n}$ is a homomorphic image of $C_{n}$ and need not be considered); $F_{4}, G_{2}$, $E_{6}, E_{7}$, with $p=2,3$ and $E_{8}$ with $p=2,3,5$.

We shall now sketch our development. We recall that an element $x$ of $G$ is regular if its centralizer $G_{x}$ has dimension equal to the rank, say $r$, of $G$, and that an element is unipotent if its eigenvalues are all 1. Relative to a Cartan decomposition of $G$ let $U$ be the maximal

1 The results are part of the author's Ph.D. thesis at the University of California at Los Angeles. The author wishes to thank Professor Robert Steinberg for his patient guidance during the preparation of this work. 
unipotent subgroup corresponding to a system $P$ of positive roots, and for each $\alpha \in P$ let $x_{\alpha}$ denote a corresponding isomorphism of $\Phi$ into $U$. We write $\pi=\left\{\alpha_{1}, \alpha_{2}, \cdots, \alpha_{r}\right\}$ for the system of simple roots and $h t(\alpha)=\sum k_{i}$ for the height of the root $\alpha=\sum k_{i} \alpha_{i}$. Each element $u$ of $U$ can be written uniquely as $u=\prod x_{\alpha}\left(t_{\alpha}\right)\left(t_{\alpha} \in \Phi\right)$, with the terms arranged according to any fixed ordering of the roots. Chevalley [1] has proved the following fundamental result.

LEMMA 1. The isomorphisms $x_{\alpha}$ can be so chosen that for $\alpha, \beta \in P$, the

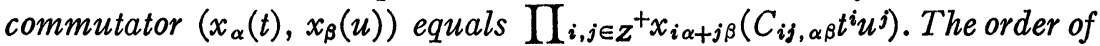
the product can be arbitrarily fixed and the $C_{i j, \alpha \beta}$ are integers which can be explicitly determined.

A typical regular unipotent element in $G$ is $\prod_{\alpha \in \pi} x_{\alpha}(1)$, an element of $U$; the product may be taken in any order. Steinberg in [4] has proved that any two regular unipotent elements are conjugate.

Lemma 2. If $x$ is a regular unipotent element of $G$, and if $x \in U$, then the centralizer $G_{x}$ of $x$ is just $U_{x}$.

For proof see [2, p. 131].

We can obtain the unique expressions in $U$ for $x u$ and $u x$, with $u=\prod x_{\alpha}\left(t_{\alpha}\right)$ as above. The parameter corresponding to each root $\alpha$ will be a polynomial in the $t_{\alpha}^{\prime}$ 's. Solving $x u=u x$ is equivalent to finding solutions to a system of polynomial equations in $q$ variables with one equation per root. Therefore $U_{x}$ can be viewed as an algebraic set in $\Phi^{q}$. For the parameter $t_{\alpha}$ associated with the root $\alpha$ we define the height of $t_{\alpha}, h t\left(t_{\alpha}\right)$, to be $h t(\alpha)$. Induction arguments and computers were used to solve the above equations. In all cases, we can describe the solution as follows: there are $r$ (the rank of $G$ ) free variables. In addition, the unique variable of height 1 may take on any value in $G F(p)$, and for $p=2, G$ of type $E_{7}$ or $E_{8}$, the unique variable of height 2 also may take on the values 0 and 1 . Hence $U_{x}$ has $p$ disjoint irreducible components except in the last two cases when there are 4 such components. The identity component, $U_{x}^{0}$, of $U_{x}$ is obtained by setting the nonfree variables mentioned above equal to zero. It follows that $U_{x} / U_{x}^{0}$ is a cyclic $p$-group. This is true for the exceptional cases since if $u \in U_{x}, u=x_{\alpha_{1}}(1) x_{\alpha_{2}}(1) \prod_{\beta} x_{\beta}\left(t_{\beta}\right)$ with $\alpha_{1}, \alpha_{2}$ simple roots, then $u^{2}=x_{\alpha_{1}+\alpha_{2}}(1) \prod_{\beta} x_{\beta}\left(t_{\beta}\right) \notin U_{x}^{0}$. It has been proved by Springer [3] that $G_{x}^{0}$ is abelian. Therefore, $U_{x}^{0}$ is abelian. The parameter corresponding to simple roots is 1 for the element $x$; thus we may take $x U_{x}^{0}$ as a generator for $U_{x} / U_{x}^{0}$. Every element $u$ in $U_{x}$ may be expressed as $x^{k} u_{0}$ with $k$ an integer and $u_{0} \in U_{x}^{0}$. It is clear now that $U_{x}$ is abelian and hence $G_{x}$ is abelian. 
If we let $U_{i}$ denote the subgroup of $U$ generated by $\left\{x_{\alpha}(t) \mid t \in \Phi\right.$, $h t(\alpha) \geqq i\}$, then as a further measure of the structure of $U_{x}$ we form the sequence of numbers $h=h_{1} \leqq h_{2} \leqq \cdots$ at which $\operatorname{dim}\left(U_{x}^{0} \cap U_{h}\right)$ decreases, i.e., of heights at which free parameters for $U_{x}^{0}$ occur. The deviations from the case $p=0$ (when the $h$ 's are well known, see, e.g., [3]) are as follows:

$C_{n}$. Here the $h$ 's are $1,3,5, \cdots, 2 n-1$ if $p=0$. All but the last are to be increased by 1 if $p=2$.

$D_{n}$. Increase the first $[(n-2) / 2] h$ 's, i.e., $1,3,5, \cdots$ by 1 .

$E_{6}$ or $G_{2}$. Increase $h_{1}$ from 1 to $p$ (which is 2 or 3 ).

$E_{7}$. Increase $h_{1}, h_{3}$ from 1,7 to 4,8 if $p=2$, and $h_{1}$ to 3 if $p=3$.

$E_{8}$. Increase $h_{1}, h_{2}, h_{4}$ from $1,7,13$ to $4,8,14$ if $p=2 ; h_{1}, h_{2}$ to 3,9 if $p=3 ; h_{1}$ to 5 if $p=5$.

$F_{4}$. Increase $h_{1}, h_{3}$ from 1,7 to 2,8 if $p=2$, and $h_{1}$ to 3 if $p=3$.

\section{BIBLIOGRAPHY} 66.

1. C. Chevalley, Sur certains groupes simples, Tôhoku Math. J. (2) 7 (1955), 14-

2. T. Springer, Some arithmetical results on semisimple Lie algebras, Inst. Hautes Etudes Sci. Publ. Math., No. 30 (1966), 115-142.

3. - A note on centralizers in semisimple group, Nederl. Akad. Wetensch. Proc. Ser. A69= Indag. Math. 28 (1966), 75-77.

4. R. Steinberg, Regular elements of semisimple algebraic groups, Inst. Hautes Etudes Sci. Publ. Math., No. 25 (1965), 49-80.

University of California at Los ANgeles and NORTHEASTERN UNIVERSITY 